Luciane Maria Oliveira Brito ${ }^{1}$

Maria Bethânia da Costa Chein ${ }^{2}$

Luiz Gustavo Oliveira Brito 3

Ângela Mirella Magalhães AmOrim ${ }^{4}$

Heitor Ricardo Cosiski Marana ${ }^{5}$

Artigo original

Palavras-chave

Autoexame

Programas de rastreamento

Conhecimentos, atitudes e práticas em

saúde

Mama/patologia

Neoplasias da mama/diagnóstico

Keywords

Self-examination

Mass screening

Health knowledge, attitudes, practice

Breast/pathology

Breast neoplasms/diagnosis

\section{Conhecimento, prática e atitude sobre o autoexame das mamas de mulheres de uma cidade do Nordeste do Brasil}

\author{
Knowledge, practice and attitude about breast self-exam from women of \\ a Northeastern municipality, Brazil
}

Resumo

OBJETIVO: avaliar o conhecimento, a atitude e a prática do autoexame das mamas (AEM) em mulheres do município de São Luís (MA) e os fatores sociodemográficos relacionados. MÉTODOS: estudo transversal, prospectivo, com amostragem por conglomerados, no qual foram incluídas 552 mulheres de 14 setores censitários em São Luís, no período de Janeiro a Setembro de 2003. O conhecimento, a atitude e a prática (variáveis dependentes) foram avaliados pela análise das respostas das mulheres em "adequado" ou "inadequado". As principais variáveis independentes foram: idade, escolaridade, renda familiar, situação conjugal e status menopausal. Foi realizado o teste do $\chi^{2}$ para a associação entre variáveis categóricas e a mensuração do Odds Ratio (OR) bruto e ajustado após análise multivariada por regressão logística. RESULTADOS: embora 1/3 da população estudada não tivesse conhecimento do AEM, o grupo de mulheres que eram informadas sobre a existência do exame possuía conhecimento $(60,9 \%)$, prática $(59,5 \%)$ e atitude (90\%) adequados. O histórico familiar para câncer de mama $(8,9 \%)$ não esteve associado a maior conhecimento e prática. A mídia mostrou-se de grande importância $(63,6 \%)$ na disseminação da informação do AEM. Após análise multivariada, a mulher com parceiro $(O R=1,9)$ apresentou maior adequação do conhecimento; o grupo de mulheres com idade maior que 50 anos $(O R=1,7)$ teve melhor atitude quanto ao $A E M$; a escolaridade maior que cinco anos $(O R=2)$ e mulher com parceiro $(O R=1,7)$ estiveram associadas à prática mais correta do AEM. CONCLUSÃO: a maioria das pacientes conhece e pratica o AEM em São Luís e a atitude delas frente ao procedimento é extremamente positiva. Houve uma grande participação da mídia na propagação da informação sobre o AEM.

\section{Abstract}

PURPOSE: to assess the knowledge, attitude and practice of breast self-examination (BSE) of women from the municipality of São Luís (MA), Brazil, and associated socio-demographic variables. METHODS: prospective and cross-sectional study, with conglomerate sampling, in which 552 women from 14 census sections of São Luís were included during the period from January to September 2003. The knowledge, attitude and practice (dependent variables) were evaluated by means of analysis of the responses of the women as "adequate" or "inadequate". The main independent variables were: age, schooling, family income and marital and menopausal status. The $\chi^{2}$ test was used to determine the association between categorical variables and the measurement of the crude/adjusted Odds Ratio (OR) after multivariate analysis by means of logistic regression. RESULTS: although 1/3 of the studied population did not know about BSE, the group of women who were informed about it showed adequate knowledge (60.9\%), practice (59.5\%) and attitude $(90 \%)$. The family history of breast cancer (8.9\%) was not associated with better knowledge and practice. The media (63.6\%) was found to be important in disseminating information about BSE. After multivariate analysis, women with a partner $(O R=1.9)$ presented more adequate knowledge; women older than 50 years $(O R=11.7)$ had a better attitude towards $\mathrm{BSE}$; women with more than five years of schooling $(O R=2)$ and with a partner $(O R=1.7)$ were associated with a more correct practice of BSE. CONCLUSION: most of the patients know and practice the BSE in São Luis and their attitude towards the procedure is extremely positive. There was a great participation of the media in the dissemination of information concerning BSE.
Correspondência:

Luciane Maria Oliveira Brito

Prédio do ILA

Praça Gonçalves Dias, $s / n, 2^{\circ}$ andar - Centro CEP 65020-240 - São Lús (MA), Brosi

Fone/Fax: (98) 3232-0286

E-mail: lucianebrito@ufma.br

Recebido

$16 / 6 / 2009$
Trabalho realizado no Departamento de Medicina III da Universidade Federal do Maranhão - UFMA - São Luís (MA), Brasil.

'Professora Associada de Ginecologia do Departamento de Medicina III da Universidade Federal do Maranhão - UFMA - São Luís (MA), Brasil.

2 Professora Adjunta de Ginecologia do Departamento de Medicina III da Universidade Federal do Maranhão - UFMA - São Luís (MA), Brasil.

${ }_{3}^{3}$ Médico Assistente e Pós-graduando (Doutorado) do Departamento de Ginecologia e Obstetrícia do Hospital das Clínicas da Faculdade de Medicina de Ribeirão Preto da Universidade de São Paulo - USP - Ribeirão Preto (SP), Brasil.

${ }^{4}$ Enfermeira do Hospital Universitário da Universidade Federal do Maranhão - UFMA - São Luís (MA), Brasil.

5 Médico Assistente do Departamento de Ginecologia e Obstetrícia do Hospital das Clínicas da Faculdade de Medicina de Ribeirão Preto da Universidade de São Paulo - USP - Ribeirão Preto (SP), Brasil. 


\section{Introdução}

A incidência de câncer de mama continua crescente e, embora a detecção precoce seja mais freqüênte e tenha havido melhoras no tratamento é, ainda, causa de significativas repercussões sobre a mulher ${ }^{1}$. No Brasil, o câncer de mama é o mais incidente em todas as regiões, exceto na região Norte, na qual perde para o câncer de colo uterino, segundo dados do Instituto Nacional do Câncer (INCA). Este estimou, para todo o Brasil, aproximadamente 49.240 casos novos em $2010^{2}$.

Não existe nenhum método isento de falhas como prevenção primária para o câncer de mama; porém, existem três estratégias de prevenção secundária para a detecção precoce: o autoexame das mamas (AEM), o exame clínico das mamas (ECM) e a mamografia bilateral (MMG), sendo esta última considerada o método de eleição de rastreio em programas populacionais pelo seu impacto sobre a mortalidade ${ }^{3}$.

Embora não existam na literatura resultados de pesquisas evidenciando uma diminuição da mortalidade pelo câncer de mama com a utilização do AEM, esta modalidade de avaliação deve ser estimulada para a detecção precoce de alterações mamárias em países em desenvolvimento e subdesenvolvidos, visto que tal diagnóstico é tardio, refletindo em estádios clínicos mais avançados ${ }^{4,5}$.

Destacam-se como vantagens do AEM a detecção de tumorações pequenas ou ainda confinadas à glândula mamária, além de ser um método conveniente, útil, sem custo e de fácil execução ${ }^{6}$. A descoberta de tumores de menor diâmetro leva a um tratamento menos mutilante e existem evidências de que medidas realizadas com o intuito de reduzir o estadiamento clínico causam benefício à sobrevida e diminuem os custos do tratamento ${ }^{6,7}$.

Contudo, menos da metade da população realiza o AEM regularmente, e alguns trabalhos realizados no Brasil investigando o conhecimento, prática e atitude sobre o AEM têm mostrado baixos percentuais desses índices $^{8-10}$. No Maranhão, a incidência de câncer de mama tem aumentado temporalmente, porém ainda permanece em segundo lugar em relação ao câncer de colo uterino ${ }^{11}$. Torna-se necessária a intervenção dos profissionais de Saúde na conscientização da mulher sobre a importância da prevenção do câncer de mama.

Devido ao desconhecimento sobre a cobertura do AEM, o nível de conhecimento e prática das pacientes sobre o mesmo e, principalmente, como essas mulheres enxergam a sua realização, o presente estudo visou determinar essas variáveis na população de São Luís (MA) e identificar fatores que poderiam influenciar tais índices.

\section{Métodos}

\section{Amostragem}

Estudo transversal, prospectivo, dividido em setores censitários, por meio de amostragem por conglomerados. Após a divisão do município em estratos urbanos rurais, utilizouse a lista ordenada desses setores no município de São Luís (MA), e foi aplicada a técnica de amostragem sistemática, cuja seleção seguiu a estratégia PPT (tamanho proporcional à população), definida pela expressão: intervalo=número de habitantes/número de setores. A pesquisa foi realizada no período de Janeiro a Setembro de 2003.

Para o cálculo do tamanho amostral, foi utilizada a fórmula $\mathrm{n}=\left(\mathrm{z} \alpha^{2} \cdot \mathrm{p} \cdot \mathrm{q}\right) \mathrm{d}^{2}$. ef, supondo-se amostragem casual simples. Nessa equação, $\mathrm{z}=$ desvio padrão do índice de confiança; $\alpha^{2}=$ nível de significância ao quadrado; p.q=porcentagem pelo qual o fenômeno ocorre; $\mathrm{d} 2$ = índice de confiança; e ef=erro amostral. O erro tipo I foi fixado em $5 \%$ e a precisão absoluta desejada, em torno da estimativa de $7,5 \%$. O produto p.q foi utilizado para se conseguir a amostra mais abrangente possível. A partir da proporção esperada de $50 \%$ da cobertura do AEM, a amostra final necessária seria de 171 mulheres. Como a amostragem foi realizada por conglomerado, foi estimado um efeito de desenho em torno de 2 para os indicadores, com um total de 342 mulheres. Considerando-se um percentual de perdas de $20 \%$, calculou-se o mínimo de 410 pacientes para este trabalho.

Sabendo-se que o município de São Luís é composto por 780 setores censitários, segundo informações do Censo 2000 do Instituto Brasileiro de Geografia e Estatística $(\mathrm{IBGE})^{12}$, foram sorteados 14 setores censitários. A partir daí, obtivemos mapas de todos esses setores, sendo suas quadras identificadas e numeradas e as três primeiras de cada setor foram catalogadas por ordem de sorteio. $\mathrm{O}$ passo seguinte foi a identificação, com letras, das esquinas das quadras sorteadas também nos mapas, sendo, depois, realizado sorteio para identificação da esquina onde começou o inquérito domiciliar, com visitas a partir desse ponto, seguindo a direção horária. Esses sorteios foram realizados por amostragem aleatória simples e para o reconhecimento in loco. Dividiu-se o número de mulheres desejadas pelo número médio de pessoas por domicílio e obteve-se o número de domicílios a serem visitados.

Antes de o projeto ser realmente efetivado, foi desenvolvido um estudo piloto com 35 mulheres sorteadas, aleatoriamente, de um setor censitário, a fim de adequarmos melhor as respostas do questionário e adaptação das entrevistadoras ao mesmo, para maior exploração e validação dos dados.

Assim, as visitas foram iniciadas no primeiro setor, na primeira quadra e na primeira esquina sorteadas, seguindo-se a direção horária, com entrevistas a mulheres 
a partir da menarca, em todos os domicílios, até um total de 40 entrevistas. Quando foram encontrados domicílios fechados, ou a mulher moradora estava ausente, retornamos três vezes e deixamos uma carta no domicílio das mesmas. Não tivemos nenhum caso de recusa ou ausência.

Quando não conseguimos obter um total de 40 entrevistas na primeira quadra, passamos para a segunda quadra e segunda esquina sorteadas na direção horária, sendo visitados todos os domicílios e, assim, sucessivamente.

\section{Variáveis dependentes e independentes}

Um questionário semiestruturado foi aplicado às pacientes. A assinatura do termo de consentimento livre e esclarecido era obrigatoriamente precedido à entrevista. Esta pesquisa teve aprovação do Comitê de Ética em Pesquisa do Hospital Universitário da Universidade Federal do Maranhão.

As variáveis sociodemográficas analisadas foram: idade, estado civil, cor, cidade, escolaridade, renda familiar, profissão, número de gestações, antecedentes familiares de câncer de mama, vícios (tabagismo e alcoolismo), atividade física e estado menstrual.

Foi feita uma análise sobre o conhecimento, a prática e a atitude das pacientes quanto ao autoexame das mamas. Conceitualmente, foram definidos: conhecimento - habilidade de recordar fatos específicos sobre determinado assunto, com comprovação científica do mesmo; prática - executar a ação sobre determinado assunto com comprovada perícia; atitude - emissão de opiniões e de ideias sobre um determinado assunto.

Considerou-se conhecimento adequado do autoexame as afirmações corretas das entrevistadas quanto à função do procedimento, maneira e frequência de realização. A atitude das mulheres foi considerada adequada quando elas consideravam o AEM um procedimento de grande importância quanto à sua realização. Quanto à prática, pedíamos à paciente que demonstrasse o autoexame por meio de gestos e como elas o faziam na frente do espelho. Caso os passos realizados fossem corretos, considerávamos a prática adequada.

Foi iniciado o processamento de dados pela revisão do questionário pelo pesquisador com correção de fichas em caso de dúvidas ou falhas nos preenchimentos.

\section{Análise estatística}

A digitação e o processamento com dupla entrada dos dados foram realizados utilizando-se o programa Epi Info $^{\circledR} 2000$ (CDC, Atlanta, USA). Para a análise estatística, foi utilizado o programa Intercooled Stata 9.0 (Stata Corporation, College Station, Texas, USA), com cálculo de médias, desvios padrão e medianas para variáveis contínuas. As variáveis binomiais tiveram seus resultados expressos em porcentagens. O teste do $\chi^{2}$ foi utilizado para o estudo da associação existente entre as diversas variáveis independentes e as três variáveis dependentes (conhecimento, atitude e prática) do autoexame das mamas. Nos casos onde o alfa era de $10 \%$, foi aprofundada essa análise para um estudo univariado. Para eliminar os fatores de confusão existentes, uma análise multivariada foi realizada por meio de um método de regressão logística (stepwise with backward elimination) para avaliar as variáveis possivelmente associadas com uma maior adequação ao conhecimento, prática e atitude do AEM, com cálculo do Odds Ratio (OR) bruto/ajustado e seus respectivos intervalos de confiança. Adotou-se uma significância estatística final de 5\%.

\section{Resultados}

Foram entrevistadas 552 mulheres dos 14 setores censitários previamente sorteados de forma aleatória. A média de idade e a mediana das mulheres eram de, respectivamente, 35,3 e 35 anos (desvio padrão de 0,7 ). As entrevistadas tinham, na sua maioria, o seguinte perfil: menor que 35 anos $(55,4 \%)$, referiam ter cor parda ou negra $(83,9 \%)$, provenientes de São Luís $(93,4 \%)$, escolaridade menor que cinco anos $(49,5 \%)$, com baixa renda familiar mensal (38,6\% com até um salário mínimo), sem emprego $(63,6 \%)$, porém possuindo função como mantenedora da casa $(20,8 \%)$ (Tabela 1$)$.

A média de idade e a mediana da menarca foram de, respectivamente, 13,1 e 13 anos (desvio padrão de 0,1); os mesmos valores para a idade da primeira gestação foram de, respectivamente, 20,8 e 20 anos (desvio padrão de $0,3)$. Quanto aos hábitos, foram relatadas baixas taxas de fumo $(8,2 \%)$ e álcool $(8,7 \%)$. Vinte e dois por cento das mulheres referiram realizar atividade física. A maioria das mulheres encontrava-se no menacme, com baixo percentual $(24,5 \%)$ de menopausadas. Quanto ao histórico reprodutivo, a maioria era de multíparas $(49,7 \%)$. Aproximadamente $9 \%$ das pacientes possuíam histórico familiar para o câncer de mama.

Foi encontrado um alto percentual de pacientes $(32,2 \%)$ que não conheciam o AEM. Contudo, das pacientes que apresentaram conhecimento $(\mathrm{n}=374)$, este era adequado na maioria $(60,9 \%) ; 23 \%$ das pacientes já haviam tido suas mamas examinadas por algum profissional de Saúde. A fonte de informação mais importante sobre o autoexame foi a mídia, seguido do médico. A atitude com relação ao AEM também foi adequada na maioria das pacientes (90\%). Quanto à prática, em mais da metade $(59,5 \%)$ foi considerada adequada. Entre as pacientes que não realizavam o AEM, os motivos principais relatados foram o esquecimento e a falta de orientação (Tabela 2).

A análise de variáveis sociodemográficas mostrou uma associação positiva entre mulheres com parceiro (OR bruto/ajustado $=1,7 / 1,9)$ e um maior conhecimento sobre o autoexame. Quanto à atitude, a idade maior que 50 anos (OR bruto/ajustado $=4,5 / 11,7)$ e a escolaridade 
Tabela 1 - Características gerais das mulheres incluídas no estudo

\begin{tabular}{|c|c|c|}
\hline Variáveis & n & $\%$ \\
\hline \multicolumn{3}{|l|}{ Faixa etária (anos) } \\
\hline $11-34$ & 306 & 55,4 \\
\hline $35-49$ & 133 & 24,1 \\
\hline 50 ou mais & 113 & 20,5 \\
\hline \multicolumn{3}{|l|}{ Escolaridade (anos) } \\
\hline $0-4$ & 273 & 49,5 \\
\hline $5-9$ & 152 & 27,5 \\
\hline 10 ou mais & 123 & 22,3 \\
\hline Ignorado & 4 & 0,7 \\
\hline \multicolumn{3}{|l|}{ Estado civil } \\
\hline Com companheiro & 208 & 37,7 \\
\hline Sem companheiro & 344 & 62,3 \\
\hline \multicolumn{3}{|l|}{ Profissc̃o } \\
\hline Com emprego & 183 & 33,2 \\
\hline Sem emprego & 351 & 63,6 \\
\hline Ignorado & 18 & 3,2 \\
\hline \multicolumn{3}{|l|}{ Renda familiar (s.m.) } \\
\hline$<1$ & 41 & 7,4 \\
\hline 1 & 172 & 31,2 \\
\hline $2-4$ & 173 & 31,3 \\
\hline $5-9$ & 55 & 9,9 \\
\hline 10 ou mais & 19 & 3,4 \\
\hline Ignorado & 92 & 16,8 \\
\hline \multicolumn{3}{|l|}{ Número de gestações } \\
\hline Nenhuma & 3 & 0,5 \\
\hline 1 & 75 & 13,6 \\
\hline 2 & 82 & 14,9 \\
\hline 3 ou mais & 192 & 34,8 \\
\hline Ignorado & 200 & 36,2 \\
\hline \multicolumn{3}{|l|}{ Estado menstrual } \\
\hline Presente & 409 & 74,1 \\
\hline Ausente & 133 & 24,1 \\
\hline Ignorado & 10 & 1,8 \\
\hline \multicolumn{3}{|c|}{ História familiar de câncer de mama } \\
\hline Sim & 49 & 8,9 \\
\hline Não & 495 & 89,7 \\
\hline Ignorado & 8 & 1,4 \\
\hline Total & 552 & 100 \\
\hline
\end{tabular}

s.m.: salário mínimo.

maior que 5 anos (OR bruto $=4,8$ ) foram fatores que estiveram relacionados a uma opinião positiva sobre o AEM. A melhor prática esteve associada à maior escolaridade $(\mathrm{OR}$ bruto/ajustado $=2,8 / 2)$ e à presença de parceiro $(\mathrm{OR}$ bruto/ajustado=2/1,7) (Tabela 3). Após realização de análise multivariada por regressão logística, mantiveram-se todos os fatores supracitados, exceto a escolaridade maior que cinco anos para uma atitude adequada.

\section{Discussão}

Verificou-se que a maioria $(67,8 \%)$ das pacientes conhecia o AEM; tais percentuais são compatíveis com
Tabela 2 - Conhecimento, atitude e prática das pacientes em relação ao autoexame das mamas

\begin{tabular}{|c|c|c|}
\hline Variáveis & $n$ & $\%$ \\
\hline \multicolumn{3}{|l|}{ Conhecimento } \\
\hline Sim & 374 & 67,8 \\
\hline Não & 178 & 32,2 \\
\hline \multicolumn{3}{|l|}{ Fonte de conhecimento* } \\
\hline Médico & 113 & 20,5 \\
\hline Enfermeiro & 17 & 3,1 \\
\hline Família & 26 & 4,7 \\
\hline Rádio, TV, jornal & 351 & 63,6 \\
\hline Outros & 34 & 6,2 \\
\hline \multicolumn{3}{|l|}{ Adequação do conhecimento** } \\
\hline Adequado & 228 & 60,9 \\
\hline Inadequado & 146 & 39,1 \\
\hline \multicolumn{3}{|l|}{ Atitude** } \\
\hline Adequada & 337 & 90 \\
\hline Inadequada & 11 & 2,9 \\
\hline Ignorado & 26 & 7,1 \\
\hline \multicolumn{3}{|l|}{ Prática** } \\
\hline Adequada & 223 & 59,5 \\
\hline Inadequada & 151 & 40,5 \\
\hline \multicolumn{3}{|c|}{ Motivos para não realizar o autoexame* } \\
\hline Falta de orientação & 38 & 20,3 \\
\hline Acha que é ainda muito nova & 32 & 17,1 \\
\hline Esquecimento & 46 & 24,6 \\
\hline Outros & 71 & 37,9 \\
\hline
\end{tabular}

outros estudos realizados em diversas cidades brasileiras. Em Goiânia ${ }^{8}$ e $\mathrm{Natal}^{13}, 75 \%$ das pacientes conheciam o AEM; em Belém ${ }^{10}$, um percentual maior $(96 \%)$ referiu ter noção sobre o AEM, índice similar a um estudo de Campinas $^{9}(95,3 \%)$. Porém, ao contrário de algumas cidades, a adequação do conhecimento foi considerada boa (60,9\%); este percentual, avaliado em Campinas ${ }^{9}$, foi de $7,4 \%$. O baixo índice também é encontrado em minorias étnicas de países desenvolvidos, como nas descendentes afro-americanas dos Estados Unidos, onde o índice de câncer de mama é alto ${ }^{14}$.

Talvez o alto percentual da mídia como fonte mais frequente de conhecimento possa ter levado à maior disseminação das informações corretas. Contudo, não se evidenciou nenhuma associação significante $\left(\mathrm{p}=0,8 ; \chi^{2}=0,02\right)$. Apesar disso, isso não substitui o dado preocupante de que $1 / 3$ das pacientes não conhece um procedimento de fácil execução. Alguns trabalhos já foram realizados no sentido de avaliar se uma intervenção educacional poderia aumentar o nível de conhecimento sobre o AEM. Uma pesquisa, comparando um grupo submetido a orientações por vídeo e a práticas em modelos de silicone sobre o AEM, com uma população controle, evidenciou uma taxa de melhora em $90 \%$ no conhecimento e prática desse grupo em relação ao $\mathrm{AEM}^{15}$. 
Tabela 3 - Adequação do conhecimento, atitude e prática das mulheres em relação ao autoexame, segundo variáveis sociodemográficas

\begin{tabular}{|c|c|c|c|c|c|c|}
\hline Variáveis & Adequado (\%) & Inadequado (\%) & $\chi^{2}$ & Valor de $\mathrm{p}$ & OR bruto (IC95\%) & OR ajustado (IC95\%) \\
\hline \multicolumn{7}{|l|}{ Conhecimento } \\
\hline Idade $>50$ anos & 57,1 & 42,9 & 0,5 & NS & - & - \\
\hline Escolaridade $>5$ anos & 57 & 43 & 1,9 & NS & - & - \\
\hline Estado civil com companheiro & 68,1 & 31,9 & 6 & 0,01 & $1,7(1,11-2,62)$ & $1,91(1,18-3,1)$ \\
\hline Renda familiar $>5$ salários mínimos & 64,9 & 35,1 & 0,3 & NS & - & - \\
\hline Com emprego & 59,1 & 40,9 & 0,1 & NS & - & - \\
\hline Idade $>50$ anos & 91,9 & 8,1 & 9,9 & $<0,01$ & $4,46(1,63-12,2)$ & $11,68(1,17-26,32)$ \\
\hline Escolaridade $>5$ anos & 94,7 & 5,3 & 7 & $<0,01$ & $4,79(1,34-17,04)$ & - \\
\hline Estado civil com companheiro & 97,3 & 2,7 & 0,2 & NS & - & - \\
\hline Renda familiar $>5$ salários mínimos & 97,2 & 2,8 & 0,08 & NS & - & - \\
\hline Com emprego & 97,7 & 2,3 & 0,07 & NS & - & - \\
\hline Escolaridade $>5$ anos & 48,8 & 51,2 & 17,3 & $<0,01$ & $2,77(1,53-3,35)$ & $2,04(1,28-3,26)$ \\
\hline Estado civil com companheiro & 69,8 & 30,2 & 10,9 & $<0,01$ & $1,99(1,32-3)$ & $1,73(1,08-2,77)$ \\
\hline Renda familiar $>5$ salários mínimos & 73,5 & 26,5 & 3,5 & NS & - & - \\
\hline Com emprego & 63,8 & 36,2 & 1,3 & NS & - & - \\
\hline Mulheres na pós-menopausa & 61,5 & 38,5 & 0,08 & NS & - & - \\
\hline
\end{tabular}

IC95\%: intervalo de confiança de $95 \%$.

A atitude quanto ao AEM foi semelhante (90\%) em relação a um estudo em Campinas ${ }^{9}(95,9 \%)$, sem mudanças no seu percentual, mesmo quando se avaliou apenas o grupo de pacientes com histórico familiar para câncer de mama. Contudo, no grupo de faixa etária mais avançada, observou-se uma maior porcentagem dessas pacientes que manifestaram uma atitude mais positiva frente ao AEM. Isso é extremamente benefício para essa população, considerando-se a maior incidência de câncer de mama nesse subgrupo. Essa tendência não foi verificada em trabalho realizado em Botucatu ${ }^{16}$. Um fator que pode ter influenciado esse resultado foi o grande número de mulheres jovens nesta pesquisa, o que poderia sugerir que, nessa faixa etária, haveria uma atenção menor frente ao tema câncer de mama.

Quanto à prática adequada do AEM, esta teve índice discretamente maior do que em mulheres de Goiânia ${ }^{8}(51 \%)$ e bem mais elevado do que em Campinas ${ }^{9}(16,7 \%)$ e em Botucatu ${ }^{16}(27 \%)$, tendo a alta escolaridade como principal fator associado após análise multivariada. Acredita-se que quanto maior for o grau de estudo, maiores serão as oportunidades de acesso aos serviços de Saúde e melhor será o conhecimento adquirido sobre métodos de prevenção. Em Goiânia ${ }^{8}$, a prática correta esteve associada a mulheres de maior escolaridade, sem emprego e com mais de 30 anos. Quanto aos motivos para a sua não realização, a maioria das mulheres respondeu que não se lembrava de realizálo, seguido de desconhecimento sobre a técnica correta, sendo esse motivo mais relatado em Belém ${ }^{10}$.
A presença de parceiro favoreceu uma maior adequação do conhecimento e prática, sendo um importante fator de proteção; todavia, a situação conjugal não apresentou associação estatisticamente significante no estudo de Goiânia $^{8}$, tampouco em Campinas ${ }^{9}$. Sabe-se que a mulher sem parceiro é fator de risco para a não realização de vários exames, como o preventivo para câncer de colo de útero, e talvez a presença do parceiro possa influenciá-la em uma maior adesão e procura por serviços de Saúde, como se observa em alguns trabalhos sobre a importância do parceiro no acompanhamento pré-natal de gestantes ${ }^{17}$.

A mídia tem um papel cada vez mais relevante na divulgação de informações sobre a história natural da doença; porém, sua importância se limita à transmissão de dados corretos, ajudando a desmitificar questões relacionadas à doença. Um estudo australiano, analisando o impacto da mídia em noticiar o diagnóstico de câncer de mama em uma artista, mostrou um aumento de 20 vezes na cobertura sobre a doença e de $40 \%$ na ida a serviços de Saúde, mantendo níveis elevados de procura das pacientes mesmo após cessação do assunto ${ }^{18}$. Em nosso trabalho, a mídia foi a principal fonte de conhecimento, de forma semelhante a outro estudo da mesma região ${ }^{10} \mathrm{e}$ diferentemente de um trabalho na região Sudeste ${ }^{9}$, onde o profissional de Saúde foi o mais prevalente.

Acredita-se que o profissional de Saúde tenha mais influência na propagação de informações relacionadas a este conteúdo, pois imagina-se que o teor científico das mesmas seja mais adequado. Em um estudo realizado 
com médicos ${ }^{19}, 98 \%$ dos pesquisados afirmaram fornecer ensinamentos sobre o AEM às suas pacientes, conhecimentos inicialmente adquiridos após cursar as disciplinas de Ginecologia no quinto ano ${ }^{20}$. Porém, as atuais recomendações de rastreamento do câncer de mama ainda vêm com pouca clareza, apresentando respostas conflitantes ${ }^{19}$. Há necessidade de se criar polos de educação continuada para esse grupo de profissionais, considerando-se os mesmos como educadores das pacientes atendidas.

Variáveis como a renda familiar e a presença de emprego não estiveram associadas à maior adequação do conhecimento ou prática nessas pacientes, ao contrário de outros estudos ${ }^{8}$. Freitas Júnior et al. concluíram que a ausência de emprego foi considerada um fator promotor de maior conhecimento, possivelmente decorrente de uma maior disponibilidade da mulher em assistir a programas educativos ${ }^{8}$. Contrariamente, em um estudo realizado na Turquia, a prática do AEM foi bem menor no grupo das mulheres mais idosas, de baixa escolaridade e do $\operatorname{lar}^{21}$, chamando a atenção para possíveis interferências culturais que possam influenciar esses índices.

A alta renda é um fator comumente associado à maior prática ${ }^{9}$. Uma limitação do nosso trabalho é o alto índice de respostas ignoradas nessa variável, o que prejudicou o estudo analítico da mesma. Outro dado que não foi passível de análise foi o número de gestações pelo mesmo motivo; porém, em trabalho prévio ${ }^{8}$, isso não mostrou associação estatística.

Por fim, constata-se que, apesar do razoável índice de conhecimento e prática em São Luís, 1/3 das mulheres não conhecia o exame, o que ressalta a necessidade de divulgação do método em todos os níveis assistenciais e em todas as camadas socioeconômicas da população, com orientações corretas sobre a técnica do AEM, a fim de que a mesma seja incorporada ao comportamento feminino.

\section{Referências}

1. Kawai M, Kuriyama S, Suzuki A, Nishino Y, Ishida T, Ohnuki K, et al. Effect of screening mammography on breast cancer survival in comparison to other detection methods: a retrospective cohort study. Cancer Sci. 2009;100(8):1479-84.

2. Brasil. Ministério da Saúde. Instituto Nacional de Câncer. Estimativas 2010: incidência de câncer no Brasil. Rio de Janeiro: INCA; 2009

3. Carlson RW, Allred DC, Anderson BO, Burstein HJ, Carter WB, Edge $S B$, et al. Breast cancer. Clinical practice guidelines in oncology. J Natl Compr Canc Netw. 2009;7(2):122-92.

4. Akhigbe AO, Omuemu VO. Knowledge, attitudes and practice of breast cancer screening among female health workers in a Nigerian urban city. BMC Cancer. 2009;9:203.

5. Cazap E, Buzaid AC, Garbino C, de la Garza J, Orlandi FJ, Schwartsmann $G$, et al. Breast cancer in Latin America: results of the Latin American and Caribbean Society of Medical Oncology/ Breast Cancer Research Foundation expert survey. Cancer. 2008; 113 (8 Suppl):2359-65.

6. Lyman $\mathrm{GH}$. Breast cancer screening: science, society and common sense. Cancer Invest. 2010;28(1):1-6.

7. Anyanwu SN. Temporal trends in breast cancer presentation in the third world. J Exp Clin Cancer Res. 2008;27:17.

8. Freitas-Júnior R, Koifman S, Santos NRM, Nunes MOA, Melo GG, Ribeiro ACG, et al. Conhecimento e prática do auto-exame de mama. Rev Assoc Med Bras. 2006;52(5):337-41.

9. Marinho LAB, Costa-Gurgel MS, Cecatti JG, Osis MD. Conhecimento, atitude e prática do auto-exame das mamas em centros de saúde. Rev Saúde Pública. 2003;37(5):576-82.

10. Monteiro APS, Arraes EPP, Pontes LB, Campos MSS, Ribeiro RT, Gonçalves REB. Auto-exame das mamas: frequência do conhecimento, prática e fatores associados. Rev Bras Ginecol Obstet. 2003;25(3):201-5.

11. Oliveira MMHN, Silva AAM, Brito LMO, Coimbra LC. Cobertura e fatores associados à não realização do exame preventivo de Papanicolaou em São Luís, Maranhão. Rev Bras Epidemiol. 2006;9(3):325-34.

12. Instituto Brasileiro de Geografia e Estatística (IBGE). Censo demográfico 2000: características da população e dos domicílios: resultados do universo. Rio de Janeiro: IBGE; 2001.

13. Davim RMB, Torres GV, Cabral MLN, Lima VM, Souza MA. Auto-exame de mama: conhecimento de usuárias atendidas no ambulatório de uma maternidade escola. Rev Latinoam Enferm. 2003;1 1(1):21-7.

14. Sadler GR, Ko CM, Cohn JA, White M, Weldon RN, Wu P. Breast cancer knowledge, attitudes, and screening behaviors among African American women: the Black cosmetologists promoting health program. BMC Public Health. 2007;7:57.

15. Lindberg NM, Stevens VJ, Smith KS, Glasgow RE, Toobert DJ. A brief intervention designed to increase breast cancer self-screening. Am J Health Promot. 2009;23(5):320-3.

16. Molina L, Dalben I, De Luca LA. Análise das oportunidades de diagnóstico precoce para as neoplasias malignas de mama. Rev Assoc Med Bras. 2003;49(2): 185-90.

17. Duarte $G$. Extensão da assistência pré-natal ao parceiro como estratégia de aumento da adesão ao pré-natal e redução da transmissão vertical de infecções. Rev Bras Ginecol Obstet. 2007;29(4):171-4.

18. Chapman S, McLeod K, Wakefield M, Holding S. Impact of news of celebrity illness on breast cancer screening: Kylie Minogue's breast cancer diagnosis. Med J Aust. 2005; 183(5):247-50.

19. Godinho ER, Koch HA. Rastreamento do câncer de mama: aspectos relacionados ao médico. Radiol Bras. 2004;37(2):91-9.

20. Aires NM, Freitas-Júnior R, Paulinelli RR, Baêta LF, Finotti MCCF, Silveira MT. Conhecimento dos métodos para diagnóstico do câncer de mama entre estudantes de medicina. Rev Bras Ginecol Obstet. 1999;21(3):133-7.

21. Maral I, Budakoglu II, Ozdemir A, Bumin MA. Behaviors toward methods of breast cancer early detection in women over 40 years in a rural region of Ankara, Turkey. J Cancer Educ. 2009;24(2):127-8. 